\title{
Efficacy and tolerability of tocilizumab in rheumatoid arthritis patients seen in daily clinical practice in Japan: results from a retrospective study (REACTION study)
}

\author{
Hisashi Yamanaka $\cdot$ Yoshiya Tanaka $\cdot$ Eisuke Inoue $\cdot$ Daisuke Hoshi $\cdot$ \\ Shigeki Momohara $\cdot$ Kentaro Hanami · Naoki Yunoue - Kazuyoshi Saito • \\ Kouichi Amano • Hideto Kameda • Tsutomu Takeuchi
}

Received: 23 July 2010/Accepted: 9 September 2010/Published online: 16 October 2010

(C) Japan College of Rheumatology 2010

\begin{abstract}
Tocilizumab, a humanized monoclonal antibody to the interleukin 6 (IL-6) receptor, was approved for use as rheumatoid arthritis (RA) therapy in Japan in 2008, but its efficacy and tolerability in daily practice has not yet been reported. We report the results of a multicenter retrospective study on the efficacy and safety of tocilizumab involving all patients $(n=229)$ who were started on tocilizumab therapy at three rheumatology institutes in Japan from April 2008 through to March 2009. Tocilizumab was infused every 4 weeks at a dose of $8 \mathrm{mg} / \mathrm{kg}$ according to the drug labeling. Among the 229 patients, 55\% concomitantly received methotrexate (MTX) and 63\% had previously received anti-tumor necrosis factor (TNF) therapy. Average disease activity score (DAS) 28 of all 229 patients significantly decreased from 5.70 to 3.25 after 24 weeks of therapy. A European League Against Rheumatism (EULAR) good response and DAS28 remission was achieved in 57.4 and $40.7 \%$ of the patients, respectively, at 24 weeks. White blood cell counts significantly decreased and liver enzymes
\end{abstract}

H. Yamanaka $(\varangle) \cdot$ E. Inoue $\cdot$ D. Hoshi · S. Momohara Institute of Rheumatology, Tokyo Women's Medical University, 10-22 Kawada-cho, Shinjuku-ku, Tokyo 162-0054, Japan e-mail: yamanaka@ior.twmu.ac.jp

Y. Tanaka $\cdot$ K. Hanami $\cdot$ N. Yunoue $\cdot$ K. Saito

The First Department of Internal Medicine, School of Medicine, University of Occupational and Environmental Health Japan, Kitakyushu, Japan

K. Amano

Division of Rheumatology and Clinical Immunology,

Department of Internal Medicine, Saitama Medical Center,

Saitama Medical University, Saitama, Japan

H. Kameda · T. Takeuchi

Division of Rheumatology, School of Medicine,

Keio University, Tokyo, Japan and total cholesterol slightly but significantly increased; however, liver enzyme levels did not increase in patients without MTX. Tocilizumab was discontinued in 47 cases (20.5\%) due to lack of efficacy (5.2\%), adverse events (11.4\%), and other reasons (3.9\%). The overall retention rate at 24 weeks was $79.5 \%$. Based on these results, we conclude that tocilizumab therapy in daily rheumatology practice appears to be highly efficacious and well tolerated among active RA patients, including the anti-TNF therapy-refractory population. Tocilizumab infusion is therefore applicable not only as an alternative approach for anti-TNF therapy-resistant patients, but also as primary biologic therapy for active RA patients.

Keywords Rheumatoid arthritis - Tocilizumab - IL-6 . Remission - Retrospective study

\section{Introduction}

The introduction of biologic agents into the rheumatoid arthritis (RA) treatment landscape has altered both therapeutic strategies as well as patient outcomes [1-3]. Indeed, the aim of medical treatment of RA patients has shifted from the short-term improvement of the quality of life (QOL) through pain relief to the long-term improvement of QOL by the prevention of joint damage and improved mortality. Tumor necrosis factor (TNF) is considered to be a major cytokine involved in RA pathogenesis, and antiTNF therapy using monoclonal antibodies or fusion proteins was the first anti-cytokine therapy to be developed for RA [4-6]. Anti-TNF therapy has achieved great success in suppressing rheumatoid inflammation and preventing the progression of joint damage in RA patients [7-9], thereby confirming that TNF indeed appears to be critically 
involved in the pathogenesis of RA synovitis. However, TNF is not the only cytokine involved in RA pathogenesis, and several candidate cytokines represent potential therapeutic targets for anti-cytokine therapy. Interestingly, the interleukin 1 (IL-1) receptor antagonist anakinra did not demonstrate a significant therapeutic effect in the treatment of RA [10], even though IL-1 is believed to be involved in the pathogenesis of RA synovitis [11], leading to the conclusion that not all cytokines are necessarily successful therapeutic targets for RA anti-cytokine therapy.

Tocilizumab, a monoclonal antibody directed against the IL-6 receptor, was developed in Japan through a collaborative effort of Osaka University and Chugai Pharmaceutical Co. (Tokyo, Japan) [12]. After a series of carefully conducted preclinical studies, tocilizumab was evaluated in RA patients, in whom it demonstrated promising efficacy [13-15]. The efficacy of tocilizumab was assessed in several different patient groups in both Japan and Western countries, and it has been shown to improve the signs and symptoms of RA patients, to suppress the radiological progression of joint damage [16], and to improve the QOL and physical disability [17]. However, the utility of tocilizumab has not been well documented in daily clinical practice outside of the clinical trial setting.

Tocilizumab was first approved as a therapy for RA in Japan in April 2008, and approximately 9,000 Japanese RA patients had received tocilizumab by the end of 2009 . Although, as mentioned above, the efficacy and safety of tocilizumab have been well established in several randomized control trials (RCTs), given the differences between the patient populations that participate in RCTs versus those treated in clinical practice, it is also necessary to evaluate the efficacy and safety of this agent in the daily clinical rheumatology practice setting. We have therefore conducted a retrospective study of tocilizumab use in daily rheumatology practice in three rheumatology institutes in Japan and analyzed factors potentially associated with tocilizumab efficacy. Here, we describe the clinical profiles of RA patients seen in daily rheumatology practice during the first 6 months of tocilizumab treatment.

\section{Patients and methods}

Patients

The study cohort consisted of all RA patients $(n=229)$ who fulfilled the classification criteria of the American College of Rheumatology [18] and who had undergone tocilizumab treatment between April 2008 (following formal approval of tocilizumab for RA) up to March 2009 at one of three major rheumatology centers in Japan: (1) the Institute of Rheumatology of the Tokyo Women's Medical
University, (2) the First Department of Internal Medicine of the School of Medicine, University of Occupational \& Environmental Health Japan, Kitakyushu, or (3) the Division of Rheumatology and Clinical Immunology, Department of Internal Medicine, Saitama Medical Center, Saitama Medical University, Saitama. All data on these patients were evaluated retrospectively.

Demographic data, including disease duration and concomitant therapy, were collected from medical charts. The following parameters were evaluated at 24 weeks after the initial tocilizumab infusion: patient-recorded 28 tender joint counts (TJC), patient-recorded 28 swollen joint count (SJC), patient's global assessment of disease activity [patient's general health $(\mathrm{GH})$ ], erythrocyte sedimentation rate (ESR), and C-reactive protein (CRP) level.

\section{Tocilizumab therapy}

Tocilizumab was infused every 4 weeks at a dose of $8 \mathrm{mg} / \mathrm{kg}$ according to the drug labeling and the Japan College of Rheumatology guidelines for tocilizumab therapy [19]. Concomitant use of methotrexate (MTX) was at the discretion of the attending physician.

\section{Therapeutic response}

Disease activity was assessed by Disease Activity Score (DAS) 28-ESR and DAS28-CRP calculated using standard formulas [20]. Disability was assessed by the Health Assessment Questionnaire Disability Index (HAQ-DI) using the original HAQ [21] or the Japanese version of HAQ [22]. The primary clinical efficacy endpoint was the decrease in DAS28-ESR from baseline to week 24; secondary endpoints included decreases in DAS28-CRP and HAQ. Response to tocilizumab therapy was also evaluated using the European League Against Rheumatism (EULAR) response criteria [23]. Changes in laboratory data were also evaluated.

Discontinuation of tocilizumab treatment

Cases in which tocilizumab therapy was discontinued were further analyzed and the causes of discontinuation evaluated.

Statistical analysis

Patient baseline characteristics were summarized using mean, standard deviation (SD), median, and percentiles for the overall patient population and for patient subgroups defined by the concomitant use of MTX and prior use of anti-TNF agents. The primary endpoint was assessed by a $t$ test. The secondary endpoints and subgroup differences of 
these endpoints were also analyzed by the $t$ test. The last observations carried forward (LOCF) method was applied to evaluate efficacy in a valid manner because data could not be obtained from patients who discontinued tocilizumab therapy. Logistic regression was used for the exploratory analysis to identify variables associated with an EULAR good response and remission at 24 weeks as defined by DAS28-ESR. All reported $P$ values are twosided and not adjusted for multiple testing. $P$ values $<0.05$ were considered to indicate statistical significance for the primary endpoint. Data were analyzed with $\mathrm{R}$ ver. 2.9.

\section{Results}

Demographic data of patients from the three institutes

Baseline characteristics of the 229 patients who received tocilizumab therapy at the three rheumatology institutes in Japan from the time of its formal authorization for use in RA through to the end of March 2009 are shown in Table 1 . Overall, $55 \%$ of patients concomitantly received MTX, while $63 \%$ had previously received anti-TNF therapy prior to tocilizumab; thus, the baseline characteristics of patients who did/did not concomitantly receive MTX and who did/did not receive prior anti-TNF therapy are also shown in Table 1. Prior biologic agents administered before entry into the study included infliximab $(85 / 229$ cases; $37 \%)$, etanercept $(94 ; 41 \%)$, and adalimumab (4; $2 \%)$. The baseline data of patients who did and did not previously receive biologic therapy were not very different; however, the data of patients who did and did not receive concomitant MTX substantially differed. Patients who did not receive MTX had a longer disease duration, higher disease activity, and a lower incidence of previous use of anti-TNF agents. It should be noted that the average dose of MTX ( $8.73 \mathrm{mg} /$ week) was relatively low compared to that administered in Western countries, since there is an official regulation concerning the maximum dose of MTX in Japan [24].

\section{Efficacy of tocilizumab}

Clinical efficacy was evaluated by DAS28-ESR, DAS28CRP, EULAR response criteria, and HAQ-DI. Among all 229 patients, DAS28-ESR decreased from $5.70 \pm 1.24$ (mean $\pm \mathrm{SD}$ ) to $3.25 \pm 1.62$, DAS28-CRP decreased from $4.96 \pm 1.19$ to $0.87 \pm 1.41$, and HAQ-DI decreased from $1.58 \pm 0.79$ to $1.33 \pm 0.87$ at week 24 of treatment, demonstrating a significant improvement in these three clinical parameters $(P<0.0001)$. Figure 1 illustrates the decrease of the DAS28-ESR and its components (TJC, SJC, ESR, and GH) together with CRP. Although the decreases were significant for all clinical markers, the decreases in CRP and ESR were the most striking. The decrease in GH was statistically significant, but to a lesser degree than that of the other parameters.

The efficacy of tocilizumab was assessed in patients who did and did not receive concomitant MTX (Fig. 2). The mean DAS28-ESR and HAQ-DI values for patients with concomitant MTX use $(n=127)$ were $5.51 \pm 1.16$ and $1.44 \pm 0.74$, respectively, at baseline and $2.89 \pm 1.50$ and $1.14 \pm 0.78$, respectively, at 24 weeks. However, the DAS28 and HAQ-DI values of patients who did not receive concomitant MTX $(n=102)$ were $5.94 \pm 1.30$ and $1.75 \pm 0.82$, respectively, at baseline and $3.71 \pm 1.67$ and $1.58 \pm 0.92$, respectively, at 24 weeks (Fig. 2b, e). At all time points after week 4, DAS28-ESR, DAS28-CRP, and HAQ-DI values were significantly lower in patients who concomitantly received MTX $(P<0.0001)$.

The efficacy of tocilizumab therapy was also compared between patients who did and did not previously receive anti-TNF therapy. Mean DAS28-ESR and HAQ-DI values in patients who previously received anti-TNF therapy $(n=144)$ were $5.72 \pm 1.25$ and $1.54 \pm 0.75$, respectively, at baseline and $3.21 \pm 1.60$ and $1.30 \pm 0.83$, respectively, at 24 weeks; in contrast, patients who did not previously receive anti-TNF therapy $(n=85)$ had values of $5.66 \pm 1.22$ and $1.64 \pm 0.85$, respectively, at baseline and $3.33 \pm 1.67$ and $1.39 \pm 0.94$, respectively, at 24 weeks (Fig. 2c, f). At all time points, no differences were observed between patients who did and did not previously receive anti-TNF therapy.

The proportions of patients who had high disease activity, moderate disease activity, low disease activity, and remission as assessed by the DAS28-ESR were 67.9, $30.4,0.9$, and $0.9 \%$, respectively, at baseline, and 14.6, 31.4, 13.3, and 40.7\%, respectively, at 24 weeks (Fig. 3). EULAR responses at 24 weeks were good in $57.4 \%$ of patients, moderate in $30.6 \%$ of patients, and absent in $12.0 \%$ of patients, as assessed by the DAS28-ESR.

Changes of categorized disease activity were compared between patients who did and did not receive concomitant MTX. Superior efficacy was observed in patients who received concomitant MTX; remission rates by the DAS28 $(<2.6)$ were $40.7 \%$ in the total patient population, $49.2 \%$ in patients who received concomitant MTX, and $30.0 \%$ in patients who did not receive concomitant MTX. In contrast, while changes in categorized disease activity were also compared between patients who did and did not previously receive anti-TNF therapy, no differences were noted.

Similarly, response to tocilizumab as determined by the EULAR response criteria was compared between patients who did and did not receive concomitant MTX and between patients who did and did not previously receive 


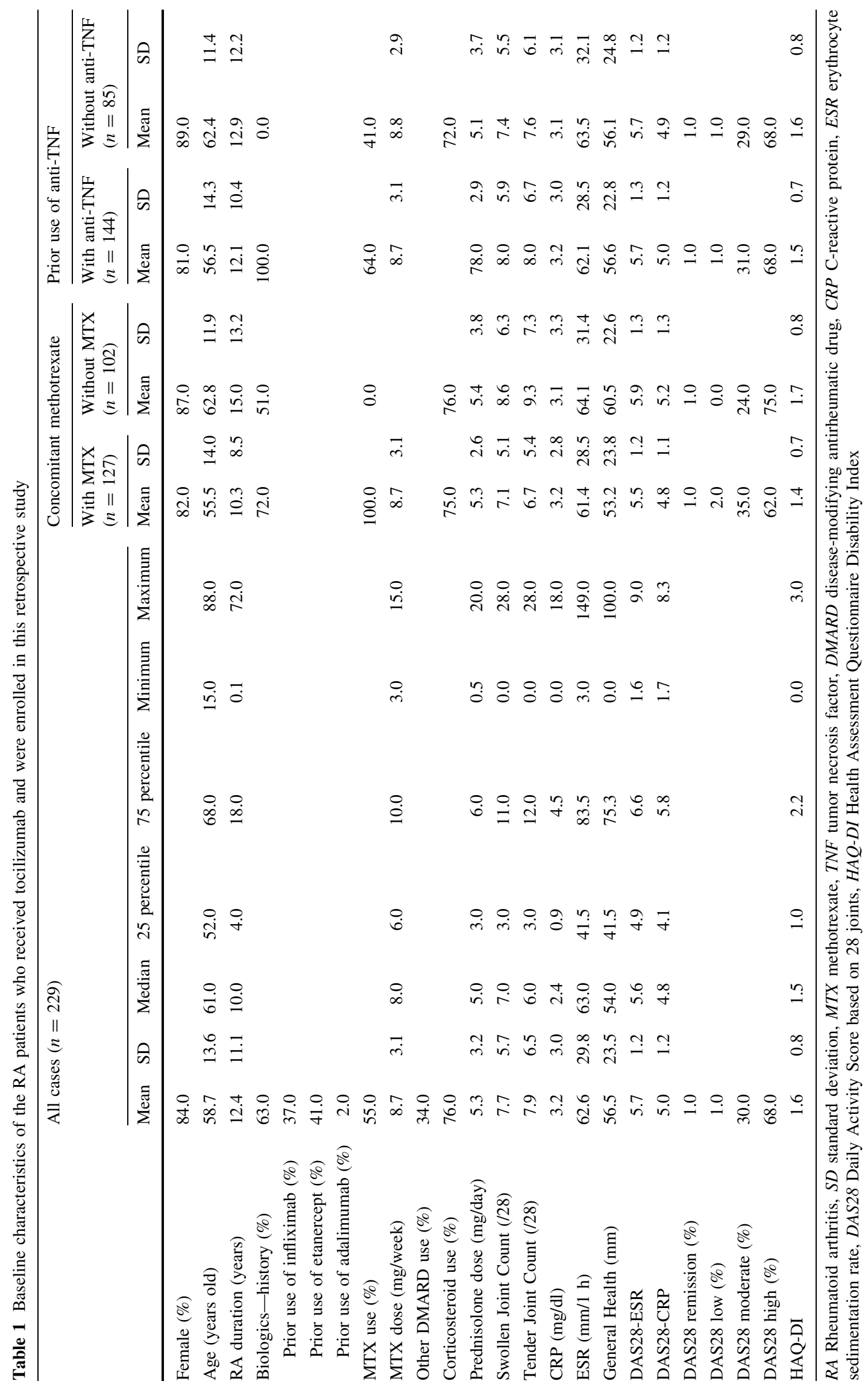



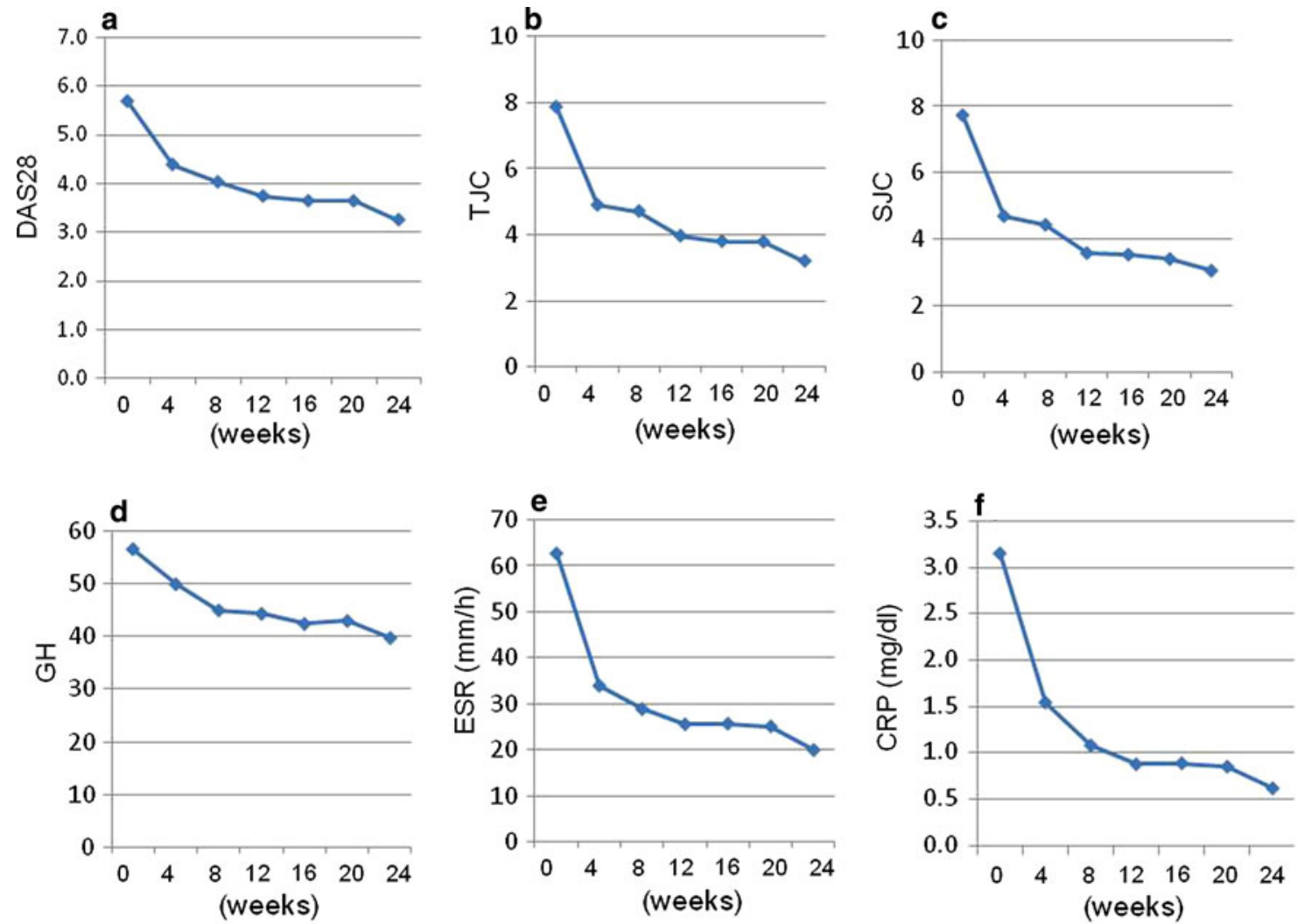

Fig. 1 Efficacy of tocilizumab infusion in inhibiting the signs and symptoms of rheumatoid arthritis (RA) patients seen in daily clinical practice. Values of the Daily Activity Score based on 28 joints $(D A S 28$; a) and its components [tender joint count $(T J C$; b), swollen joint count $(S J C ; \mathbf{c})$, good health $(G H ; \mathbf{d})$, and erythrocyte sedimentation

anti-TNF therapy. While higher efficacy was observed in patients who received concomitant MTX, prior use of antiTNF agents did not appear to influence the EULAR response.

Demographic factors related to clinical efficacy of tocilizumab

Logistic regression analysis of factors associated with a good EULAR response at 24 weeks $(n=120)$ versus moderate or no response $(n=89)$ was conducted using gender, age, disease duration, prior use of anti-TNF agent, MTX dose, prednisolone dose, HAQ at baseline and DAS28 at baseline as explanatory variables. The analysis revealed that younger age was independently associated with a good EULAR response.

Similarly, the same analysis of factors associated with remission at 24 weeks $(n=92)$ versus no remission $(n=137)$ indicated that younger age and lower DAS28 values were independently associated with remission. No other factors appeared to have a statistically significant predictive value for remission. rate $(E S R ; \mathbf{e})]$ together with C-reactive protein $(C R P)$ levels (f) from baseline to 24 weeks after the initiation of tocilizumab therapy are shown. Data were analyzed by the last observations carried forward (LOCF) method. Values were significantly lower than baseline values at all time points after 4 weeks of treatment $(P<0.00001)$

Laboratory data after initiation of tocilizumab

Laboratory data were regularly monitored throughout the 24 weeks of tocilizumab therapy (Table 2). In terms of those laboratory values used to measure disease activity, CRP levels, ESR, and matrix metalloproteinase-3 (MMP-3) levels had significantly decreased by 24 weeks, while hemoglobin $(\mathrm{Hb})$ levels had significantly increased.

With respect to laboratory parameters used to monitor safety, total cholesterol (TC) levels and liver enzyme [aspartate aminotransferase (AST) and alanine aminotransferase (ALT)] levels slightly but significantly increased, while white blood cell (WBC) counts significantly decreased. However, liver enzyme levels did not increase significantly in patients who did not concomitantly receive MTX. No clinically relevant safety findings were reported even in patients with elevated liver enzyme levels.

Discontinuation of tocilizumab therapy

A Kaplan-Meier plot of tocilizumab discontinuation is shown in Fig. 4. Tocilizumab was discontinued during the 

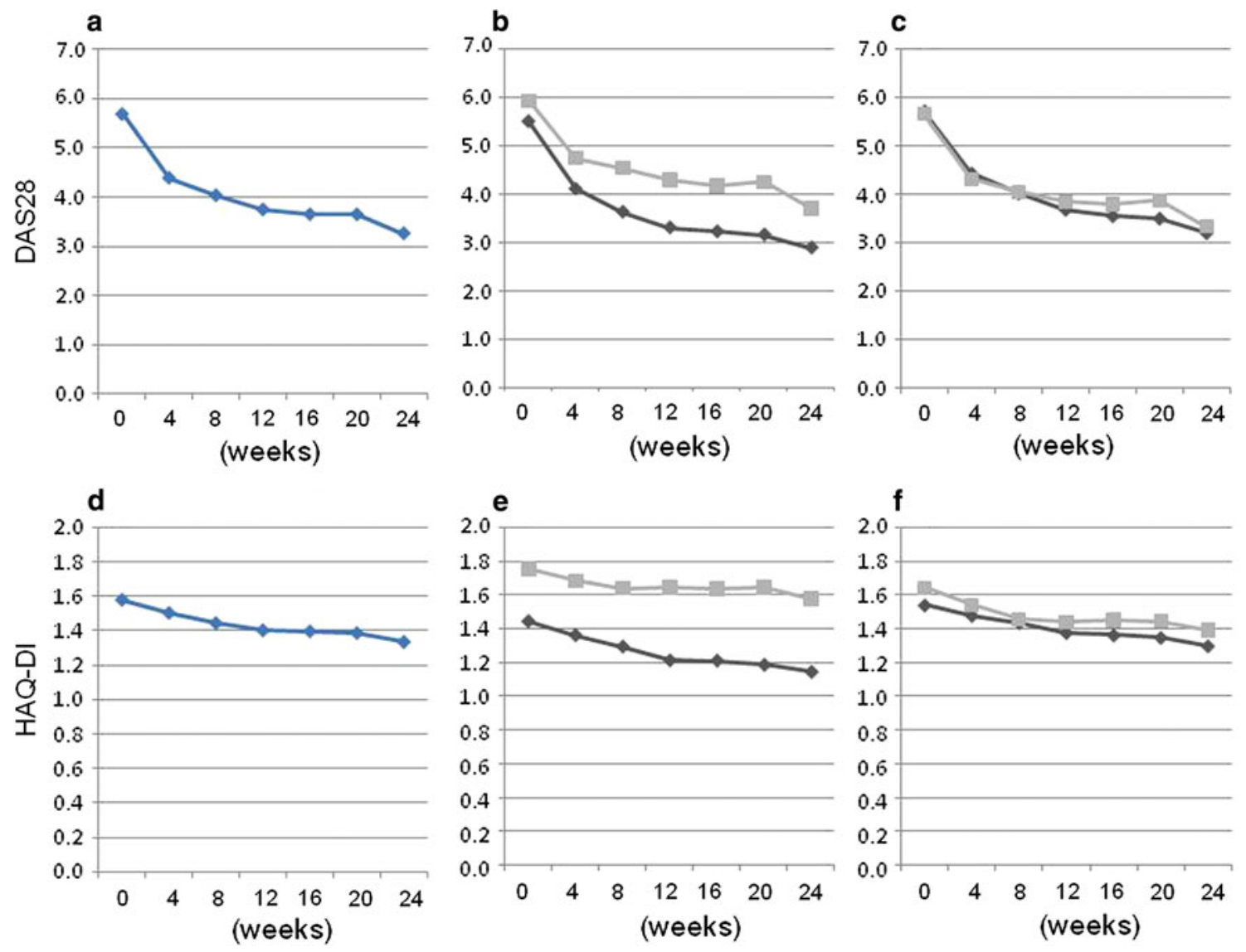

Fig. 2 Efficacy of tocilizumab infusion in inhibiting the signs and symptoms of RA patients in daily clinical practice. Values of DAS28 (a-c) and HAQ-DI (d-f) from baseline to 24 weeks after initiation of tocilizumab therapy are shown: a, d all 229 cases, b, e patients who concomitantly received methotrexate (MTX; filled diamond, $n=127$ ) or did not receive MTX (filled square, $n=102$ ), c, f patients who previously received anti-tumor necrosis factor (TNF) therapy (filled diamond, $n=144$ ) or did not previously receive anti-

24-week observation period in $47(20.5 \%)$ of the 229 patients due to a lack of efficacy (12 patients, 5.2\%), adverse events (26 patients, $11.4 \%$ ), and other reasons (9 patients, $3.9 \%$ ) - and continued in $79.5 \%$ of patients. The retention rate at 24 weeks was $80.8 \%$ in patients who concomitantly received MTX, $74.4 \%$ in those who did not concomitantly receive MTX $(P=0.197$ by log-rank test $)$, $77.6 \%$ in those who previously received anti-TNF therapy, and $78.5 \%$ in those who did not previously receive antiTNF therapy $(P=0.892$ by log-rank test). Adverse events responsible for the discontinuation of tocilizumab included six cases of pneumonia, three cases of cardiac failure and concomitant disease aggravation, two cases of hepatic failure (liver enzyme elevation), interstitial pneumonia, skin ulcer and cerebral hemorrhage, and one case of anaphylaxis, chest pain, myocardial infarction, breast cancer, leucopenia, and phlegmon (Table 3).
TNF therapy (filled square, $n=85$ ). Data were analyzed by the LOCF method. a, d At all time points after 4 weeks, values are significantly decreased from baseline $(P<0.00001), \mathbf{b}$, e at all time points after 4 weeks, values of patients who concomitantly received MTX are significantly lower than those of patients who did not $(P<0.00001), \mathbf{c}, \mathbf{f}$ at all timepoints after 4 weeks, values did not differ between patients who did and did not previously receive antiTNF therapy

\section{Discussion}

This study was conducted to determine the efficacy of tocilizumab therapy and to identify other factors associated with the effectiveness of tocilizumab therapy in Japanese RA patients receiving treatment in a university hospital outpatient setting at three rheumatic disease institutions. A study of the efficacy of tocilizumab in daily practice in a study group in the Kyushu area demonstrated the significant efficacy of this new biologic [25]. Many investigator-oriented studies of tocilizumab are currently being conducted in Japan, and more data should be available in the near future.

During the study period, only three anti-TNF therapies (infliximab, etanercept, and adalimumab) were available to treat RA in Japan; no other biologic agents, including abatacept and rituximab, was available. Tocilizumab was 
Fig. 3 Efficacy of tocilizumab infusion in inhibiting the signs and symptoms of RA patients seen in daily clinical practice. Disease activity as assessed by DAS28 values at baseline and after 24 weeks of tocilizumab therapy is shown: a all 229 cases, b patients who concomitantly received MTX $(n=127)$, c patients who did not concomitantly receive MTX $(n=102)$, d patients who previously received anti-TNF therapy $(n=144)$, e patients who did not previously receive anti-TNF therapy $(n=85)$. Disease activity was categorized as high (DAS28 $>5.1)$, moderate $(3.2 \leq$ DAS $28 \leq 5.1)$, low $(2.6 \leq$ DAS2 $<3.2)$ and remission (DAS28 < 2.6) from the top column. Data were analyzed by the LOCF method
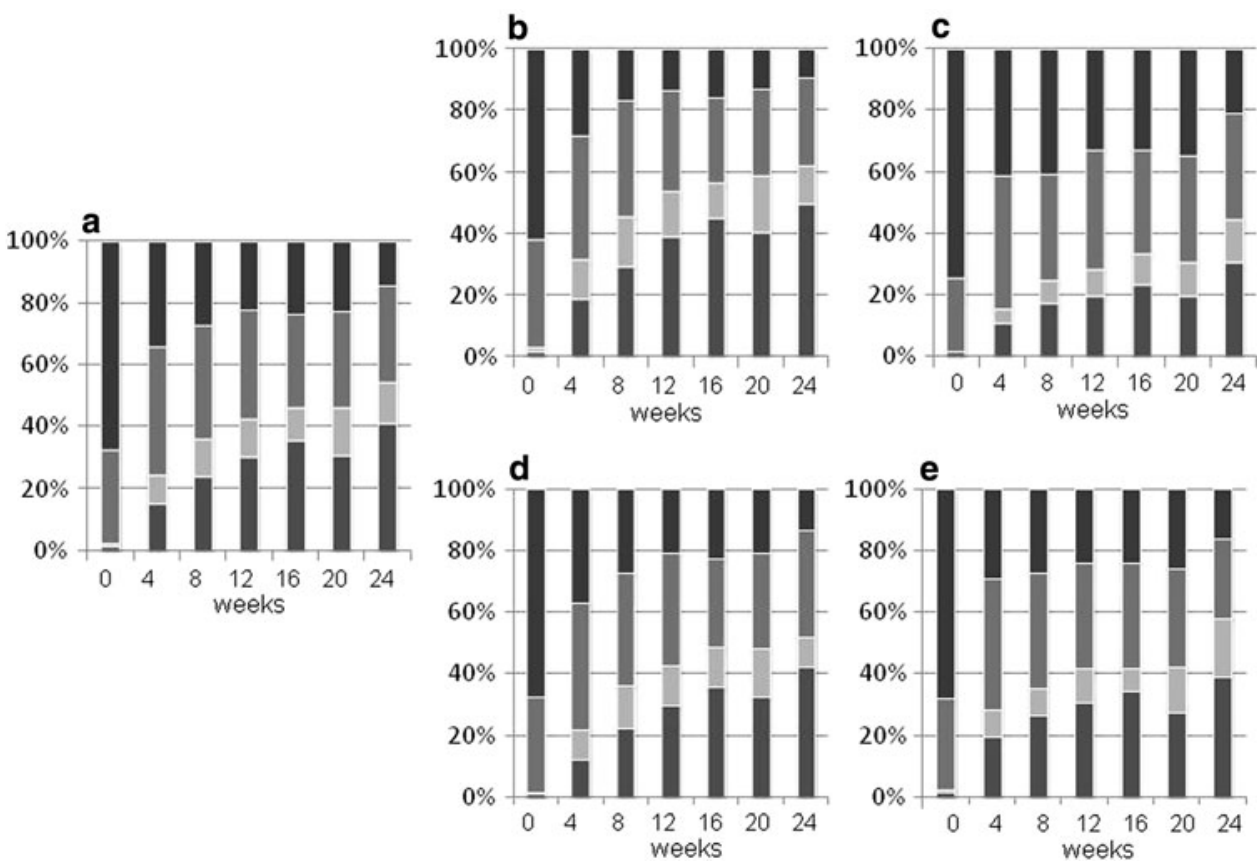

first approved in Japan in April 2008, and results from clinical studies on its efficacy and safety in RA patients seen in daily rheumatology practice outside the clinical trial setting are quite important for evaluation purposes.

All of the patients enrolled in this study were essentially MTX-resistant, two-thirds were anti-TNF therapy-resistant, and the average DAS28 at baseline was 5.70. Consequently, these patients had severe RA. Based on our results, tocilizumab therapy was highly efficacious in these RA patients seen in daily clinical practice, and the overall remission rate at 24 weeks was as high as $40.7 \%$.

It is difficult to compare efficacy in clinical studies with strict inclusion and exclusion criteria to that observed in daily clinical practice. The Japan College of Rheumatology provides a guideline for tocilizumab use [19] to which most rheumatologists comply; however, the regulations of this guideline are much less stringent compared to the inclusion and exclusion criteria of clinical studies. Even after these differences are taken into consideration, the efficacy of tocilizumab demonstrated in our study is comparable to or even higher than those reported in clinical studies. The remission rates in our study were $40.7 \%$ in the overall study population, $49.2 \%$ in patients who concomitantly received MTX, and $30.0 \%$ in patients who did not concomitantly receive MTX, while remission rates in previously reported clinical studies ranged from $27 \%$ in the OPTION study [17] to $59 \%$ in the SAMURAI study [10]. The tocilizumab-induced remission rate in the SAMURAI study was remarkably high, most likely because this study was conducted in early RA patients whose average disease duration was 2 years [10]. In contrast, the average disease duration of patients in our study was 12.4 years. Since tocilizumab directly reduces acute-phase reactants, such as CRP and ESR [26], it is not surprising that decreases in the ESR and CRP levels were among the most prominently changed DAS28 components, as shown in Fig. 1. However, other factors, including TJC, SJC, and GH, were also significantly decreased; thus, the higher remission rates observed in this study must not have been solely dependent on the potent suppression of acute-phase reactants by tocilizumab.

Concomitant use of MTX resulted in a rapid and sustained response to tocilizumab, even though the average MTX dose was relatively low (average at baseline: $8.7 \mathrm{mg}$ / week) compared to that used in Western countries. Government regulations have limited the maximum dose of MTX to $8 \mathrm{mg} /$ week in Japan; however, many rheumatologists prescribe higher MTX doses off-label [24, 27]. It is remarkable that such low-dose MTX potentiates tocilizumab action in severe RA patients. The differences in baseline characteristics between patients who did and did not concomitantly receive MTX may have accounted for this result, since patients who received MTX had a longer disease duration, higher disease activity, and lower usage of anti-TNF therapies. It is likely that tocilizumab was prescribed in combination with MTX in patients who had a lower risk of adverse events and as monotherapy in patients with a higher risk. Due to the observational nature of this study, safety issues associated with combined tocilizumab/ MTX were not sufficiently evaluated; however, considering the high retention rate (Fig. 4) and laboratory profiles (Table 2) associated with tocilizumab therapy with or without MTX, the addition of MTX does not appear to significantly increase the risk of adverse events. As the 


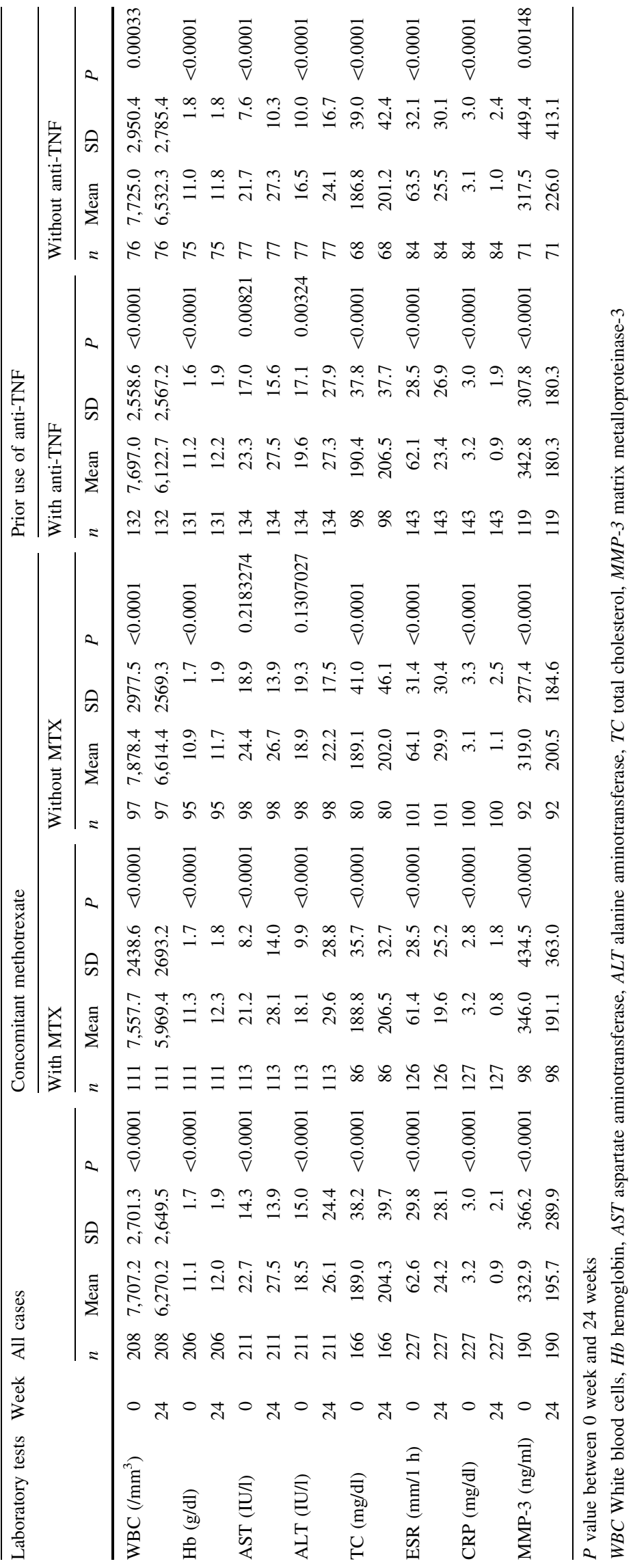


Fig. 4 Kaplan-Meier curve of the retention rate of tocilizumab therapy. a Tocilizumab retention rate in all 229 cases. b Tocilizumab retention rates by cause of discontinuation. c Tocilizumab retention rates in patients who did $(n=127)$ and did not $(n=102)$ receive concomitant MTX.

d Tocilizumab retention rates in patients who did $(n=144)$ and did not $(n=85)$ previously receive anti-TNF therapy
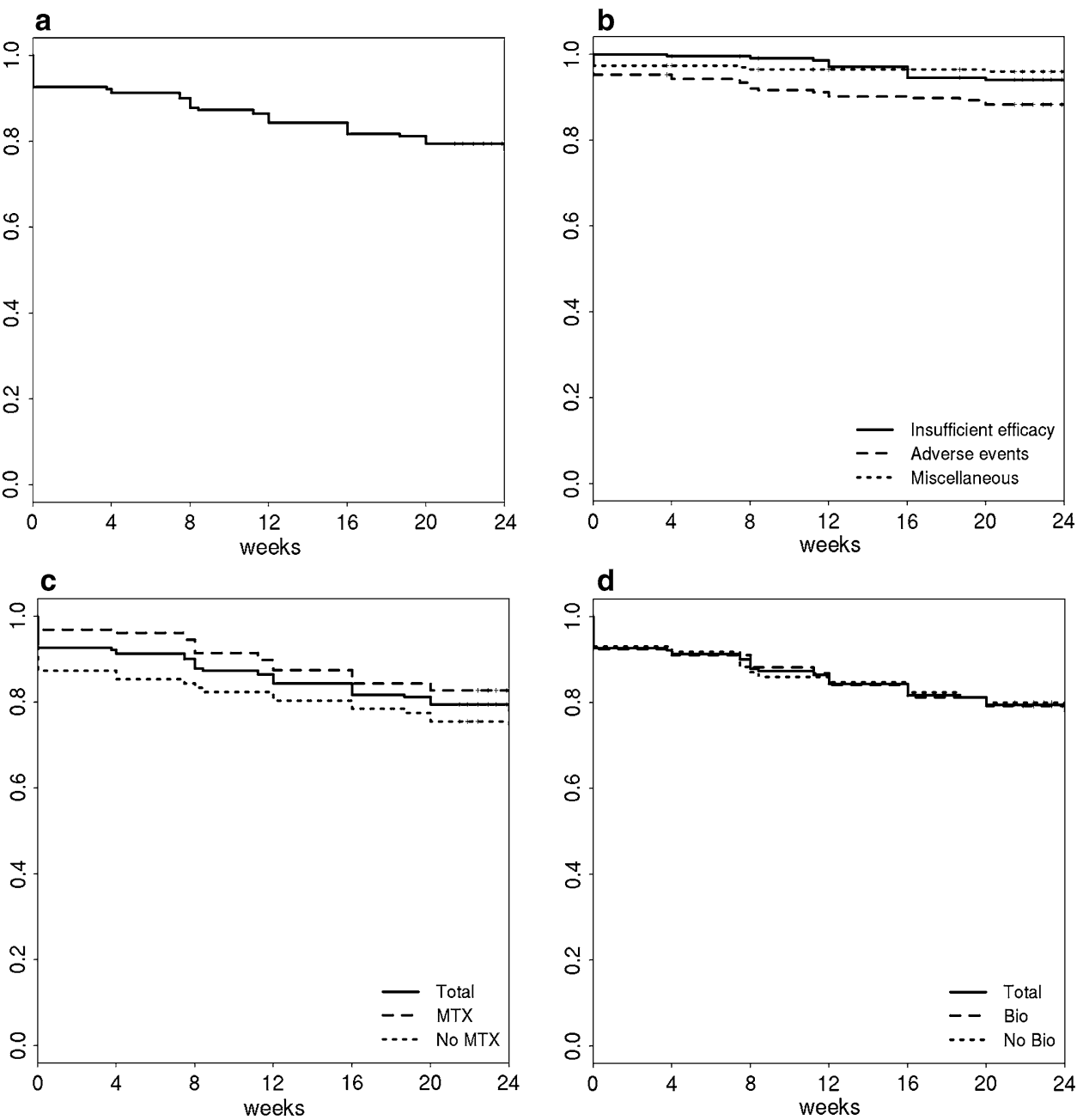

addition of MTX to the therapeutic regimen may enhance the efficacy of tocilizumab, concomitant use of MTX is recommended with tocilizumab therapy in patients in whom safety is not a concern.

An interesting finding was the lack of any differences in tocilizumab efficacy in patients who did or did not previously receive anti-TNF therapy, suggesting that clinical response to tocilizumab therapy is independent of the prior use of anti-TNF agents. This result is inconsistent with the report of Nakashima et al. [25], in which the authors reported a higher efficacy of tocilizumab in biologic naïve patients. It is highly possible that the definition of MTXrefractory or anti-TNF refractory differs between rheumatologists. Although the baseline DAS28-ESR values in our study (5.7) and that of Nalashima et al. (5.5) were comparable, the average dose of MTX at baseline was higher in our study ( $8.7 \pm 3.1$ vs. $6.8 \pm 2.1 \mathrm{mg} /$ week). This higher dose means that the definition of MTX-refractory was more stringent in our study than in that of Nakashima et al. and that our RA patients had more active disease. It is reasonable to assume that the severity of RA in patients would affect the clinical outcome after tocilizumab therapy.

Clinical data on rituximab and abatacept demonstrate that both of these agents reduce disease activity in patients with inadequate responses to anti-TNF therapy. However, the remission rates of these non-TNF biologic agents in anti-TNF-naïve or -refractory populations differ. For abatacept, the remission rate in MTX-resistant patients (AIM study) was $14.8 \%$ [28], while that in anti-TNF therapy-refractory patients (ATTAIN study) was $10.0 \%$ [29]. In the case of rituximab, the American College of Rheumatology (ACR) $70 \%$ rate in MTX-resistant patients (DANCER study) was $20 \%$ [30], and that in anti-TNF therapy-refractory patients (REFLEX study) was $12 \%$ [31]. In contrast to rituximab and abatacept, remission rates observed in tocilizumab clinical studies were quite similar between MTX-resistant patients (OPTION, 27\% [17]; TOWARD, 30\% [32]) and anti-TNF therapy-refractory patients (RADIATE, 30\% [33]). Although it may not be 
Table 3 Cause of tocilizumab discontinuation

\begin{tabular}{ll}
\hline Cause of discontinuation & Number of cases/events \\
\hline Lack of efficacy & 12 cases $(5.2 \%)$ \\
Adverse events & 26 cases $(11.4 \%)$ \\
Pneumonia & 6 \\
Heart failure & 3 \\
Worsening comorbidity & 3 \\
Liver damage & 2 \\
Interstitial pneumonia & 2 \\
Skin ulcer & 2 \\
Cerebellar hemorrhage & 2 \\
Anaphylaxis & 1 \\
Chest pain & 1 \\
Myocardial infarction & 1 \\
Leukopenia & 1 \\
Breast cancer & 1 \\
Phlegmon & 1 \\
Miscellaneous & 9 cases $(3.9 \%)$ \\
Unknown & 4 \\
Moving & 3 \\
Patient's will & 2 \\
\hline Total & 47 cases $(20.5 \%)$ \\
\hline
\end{tabular}

appropriate to compare the results of clinical trials conducted in these different patient population, it is interesting to realize that the results from tocilizumab clinical studies have been fairly consistent despite the patient population.

TNF is widely accepted as an important cytokine [4], and it is known to induce IL-6 activity [34, 35]; however, IL-6 activity is not totally dependent on TNF stimulation $[1,36,37]$. The results of our study also suggest that tocilizumab has mechanisms of action that are unique from those of anti-TNF agents. As such, TNF represents an important therapeutic option for anti-TNF agent nonresponders as well as disease-modifying antirheumatic drug (DMARD) non-responders.

In an attempt to identify predisposing factors for tocilizumab efficacy, we analyzed factors associated with remission using the demographic characteristics of our RA patients as the explanatory variables for logistic regression. The results of this analysis revealed that a low DAS28 at baseline and a younger age were associated with the induction of remission. Although patients at an earlier disease stage have been reported to be more sensitive to anti-rheumatic treatments [38], disease duration in our study did not appear to be correlated with remission. Since this study was conducted as a retrospective analysis of data collected in daily practice, many confounding factors could have potentially influenced the data. However, considering the high remission rate observed in the SAMURAI study
(59\%) [16], which was conducted in early RA patients in Japan, tocilizumab may prove to be most efficacious in younger patients and/or in those with early-stage disease.

Since IL-6 has pleiotropic actions, some of which are inconsistent with TNF actions, the inhibition of IL-6 action by tocilizumab may cause unexpected adverse reactions. Thus, the safety profile associated with tocilizumab needs to be carefully evaluated. With respect to laboratory parameters, decreased WBC counts and increased liver enzyme levels and/or serum cholesterol levels have been reported to be associated with tocilizumab use [15, 39]. However, in our study, although significant WBC count decreases and AST/ALT and TC increases were observed, these changes were tolerable (Table 2). Only two patients discontinued tocilizumab due to hepatic disorders (Table 3), and the liver enzyme levels returned to normal after the discontinuation of tocilizumab therapy. Interestingly, liver enzyme levels significantly increased in patients who concomitantly received MTX, but not in those who did not receive MTX, which is consistent with the findings of previous studies [16, 17, 32, 33]. Among 47 patients who discontinued tocilizumab, 12 did so due to insufficient efficacy, while 26 discontinued because of adverse events. Among the latter, six patients discontinued due to pneumonia. Pneumonia was the most frequent adverse event observed in previous Japanese post-marketing surveillance (PMS) evaluation of infliximab [40] and etanercept [27], which is consistent with the results of our study. However, it should be noted that susceptibility to infection is an important concern not only in association with anti-TNF therapies but also with the anti-IL-6 activity of tocilizumab.

The safety profile of tocilizumab therapy was recently extensively investigated in Japan using an all-case registered PMS study conducted by Chugai Pharmaceutical under the auspices of the regulatory authority of the Japanese government, with effective suggestions from the subcommittee of the Japan College of Rheumatology. A total of 9,000 cases were registered from April 2008 to December 2009, and an interim analysis is currently being conducted. The final analysis is expected to describe the safety profile of and risk factors associated with tocilizumab therapy on a large scale.

In conclusion, this REACTION study confirmed the efficacy of tocilizumab in Japanese patients with RA. Tocilizumab was also well tolerated, and the retention rate at 24 weeks was $79.5 \%$. The promising efficacy of tocilizumab in improving measures of disease activity and preventing progression of this disabling disease has allowed this agent to become one of the critical advances in the management of RA in recent years. The present data should facilitate a more efficacious use of this expensive biological agent in daily rheumatology practice, not only in Japan but also in many countries throughout the world. 
Acknowledgments The authors thank all medical staff in the three institutions for providing data. This work was supported partly by a Research Grant-In-Aid for Scientific Research by the Ministry of Health, Labor and Welfare of Japan, the Ministry of Education, Culture, Sports, Science and Technology of Japan, and the University of Occupational and Environmental Health, Japan.

Conflict of interest None of authors are industry employees. HY has received lecture fees from Abbott, Bristol-Meyers, Chugai Pharma, Eisai Pharma, F. Hoffmann-La Roche Ltd, Mitsubishi-Tanabe Pharma, Takeda Pharmaceutical Co Ltd, and Wyeth. YT has received consultant fees from Mitsubishi-Tanabe Pharma and Pfizer Inc, and lecture fees from Mitsubishi-Tanabe Pharma, Takeda Pharmaceutical Co Ltd, Abbott, Eisai Pharma, and Chugai Pharma. TT has received lecture fees from Abbott, Brystol-Meyers, Chugai Pharma, Eisai Pharma, Mitsubishi-Tanabe Pharma, Takeda Pharmaceutical Co Ltd., Janssen Pharmaceutical KK, and Wyeth.

\section{References}

1. Firestein GS. Evolving concepts of rheumatoid arthritis. Nature. 2003;423:356-61.

2. Weaver AL. The impact of new biologicals in the treatment of rheumatoid arthritis. Rheumatology (Oxford) 2004;43[Suppl 3]:17-23.

3. Keystone EC. Clinical implications of understanding radiographic findings in relation to clinical outcomes in rheumatoid arthritis. J Rheumatol Suppl. 2009;82:11-6.

4. Feldmann M, Brennan FM, Maini RN. Role of cytokines in rheumatoid arthritis. Annu Rev Immunol. 1996;14:397-440.

5. Elliott MJ, Maini RN, Feldmann M, et al. Randomised doubleblind comparison of chimeric monoclonal antibody to tumour necrosis factor alpha (cA2) versus placebo in rheumatoid arthritis. Lancet. 1994;344:1105-10.

6. Moreland LW, Baumgartner SW, Schiff MH, et al. Treatment of rheumatoid arthritis with a recombinant human tumor necrosis factor receptor (p75)-Fc fusion protein. $\mathrm{N}$ Engl $\mathrm{J}$ Med. 1997;337:141-7.

7. Smolen JS, Han C, Bala M, et al. Evidence of radiographic benefit of treatment with infliximab plus methotrexate in rheumatoid arthritis patients who had no clinical improvement: a detailed subanalysis of data from the anti-tumor necrosis factor trial in rheumatoid arthritis with concomitant therapy study. Arthritis Rheum. 2005;52:1020-30.

8. Klareskog L, van der Heijde D, de Jager JP, et al. Therapeutic effect of the combination of etanercept and methotrexate compared with each treatment alone in patients with rheumatoid arthritis: double-blind randomised controlled trial. Lancet. 2004;363:675-81.

9. Emery P, Breedveld FC, Hall S, et al. Comparison of methotrexate monotherapy with a combination of methotrexate and etanercept in active, early, moderate to severe rheumatoid arthritis (COMET): a randomised, double-blind, parallel treatment trial. Lancet. 2008;372:375-82.

10. Kalliolias GD, Liossis SN. The future of the IL-1 receptor antagonist anakinra: from rheumatoid arthritis to adult-onset Still's disease and systemic-onset juvenile idiopathic arthritis. Expert Opin Investig Drugs. 2008;17:349-59.

11. Abramson SB, Amin A. Blocking the effects of IL-1 in rheumatoid arthritis protects bone and cartilage. Rheumatology (Oxford). 2002;41:972-80.

12. Kishimoto $T$. Interleukin-6: from basic science to medicine40 years in immunology. Annu Rev Immunol. 2005;23:1-21.
13. Nishimoto N, Yoshizaki K, Maeda K, et al. Toxicity, pharmacokinetics, and dose-finding study of repetitive treatment with the humanized anti-interleukin 6 receptor antibody MRA in rheumatoid arthritis. Phase I/II clinical study. J Rheumatol. 2003;30: 1426-35.

14. Nishimoto N, Yoshizaki K, Miyasaka N, et al. Treatment of rheumatoid arthritis with humanized anti-interleukin-6 receptor antibody: a multicenter, double-blind, placebo-controlled trial. Arthritis Rheum. 2004;50:1761-9.

15. Maini RN, Taylor PC, Szechinski J, et al. Double-blind randomized controlled clinical trial of the interleukin- 6 receptor antagonist, tocilizumab, in European patients with rheumatoid arthritis who had an incomplete response to methotrexate. Arthritis Rheum. 2006;54:2817-29.

16. Nishimoto N, Hashimoto J, Miyasaka N, et al. Study of active controlled monotherapy used for rheumatoid arthritis, an IL-6 inhibitor (SAMURAI): evidence of clinical and radiographic benefit from an $\mathrm{x}$ ray reader-blinded randomised controlled trial of tocilizumab. Ann Rheum Dis. 2007;66:1162-7.

17. Smolen JS, Beaulieu A, Rubbert-Roth A, et al. Effect of interleukin-6 receptor inhibition with tocilizumab in patients with rheumatoid arthritis (OPTION study): a double-blind, placebocontrolled, randomised trial. Lancet. 2008;371:987-97.

18. Arnett FC, Edworthy SM, Bloch DA, et al. The American Rheumatism Association 1987 revised criteria for the classification of rheumatoid arthritis. Arthritis Rheum. 1988;31:315-24.

19. Koike R, Harigai M, Atsumi T, et al. Japan College of Rheumatology 2009 guidelines for the use of tocilizumab, a humanized anti-interleukin-6 receptor monoclonal antibody, in rheumatoid arthritis. Mod Rheumatol. 2009;19:351-7.

20. Prevoo ML, van 't Hof MA, Kuper HH, et al. Modified disease activity scores that include twenty-eight-joint counts. Development and validation in a prospective longitudinal study of patients with rheumatoid arthritis. Arthritis Rheum. 1995;38:44-8.

21. Fries JF, Spitz P, Kraines RG, Holman HR. Measurement of patient outcome in arthritis. Arthritis Rheum. 1980;23:137-45.

22. Matsuda Y, Singh G, Yamanaka H, et al. Validation of a Japanese version of the Stanford Health Assessment Questionnaire in 3,763 patients with rheumatoid arthritis. Arthritis Rheum. 2003;49:784-8.

23. van Gestel AM, Prevoo ML, van 't Hof MA, et al. Development and validation of the European League Against Rheumatism response criteria for rheumatoid arthritis. Comparison with the preliminary American College of Rheumatology and the World Health Organization/International League Against Rheumatism Criteria. Arthritis Rheum. 1996;39:34-40.

24. Yamanaka $\mathrm{H}$, Inoue $\mathrm{E}$, Tanaka $\mathrm{E}$, et al. Influence of methotrexate dose on its efficacy and safety in rheumatoid arthritis patients: evidence based on the variety of prescribing approaches among practicing Japanese rheumatologists in a single institute-based large observational cohort (IORRA). Mod Rheumatol. 2007;17: 98-105.

25. Nakashima $\mathrm{Y}$, Kondo M, Harada $\mathrm{H}$, Horiuchi $\mathrm{T}$, Ishinishi $\mathrm{T}$, Jojima H, Kuroda K, Miyahara H, Nagamine R, Nakashima H, Otsuka T, Saikawa I, Shono E, Suematsu E, Tsuru T, Wada K, Iwamoto Y. Clinical evaluation of tocilizumab for patients with active rheumatoid arthritis refractory to anti-TNF biologics: tocilizumab in combination with methotrexate. Mod Rheumatol. 2010;343-52.

26. Kishimoto T. Interleukin-6: discovery of a pleiotropic cytokine. Arthritis Res Ther. 2006;8[Suppl 2]:S2.

27. Koike $\mathrm{T}$, Harigai $\mathrm{M}$, Inokuma $\mathrm{S}$, et al. Postmarketing surveillance of the safety and effectiveness of etanercept in Japan. J Rheumatol. 2009;36:898-906.

28. Kremer JM, Genant HK, Moreland LW, et al. Effects of abatacept in patients with methotrexate-resistant active rheumatoid arthritis: a randomized trial. Ann Intern Med. 2006;144:865-76. 
29. Genovese MC, Becker JC, Schiff M, et al. Abatacept for rheumatoid arthritis refractory to tumor necrosis factor alpha inhibition. N Engl J Med. 2005;353:1114-23.

30. Emery P, Fleischmann R, Filipowicz-Sosnowska A, et al. The efficacy and safety of rituximab in patients with active rheumatoid arthritis despite methotrexate treatment: results of a phase IIB randomized, double-blind, placebo-controlled, dose-ranging trial. Arthritis Rheum. 2006;54:1390-400.

31. Cohen SB, Emery P, Greenwald MW, et al. Rituximab for rheumatoid arthritis refractory to anti-tumor necrosis factor therapy: Results of a multicenter, randomized, double-blind, placebo-controlled, phase III trial evaluating primary efficacy and safety at twenty-four weeks. Arthritis Rheum. 2006;54:2793-806.

32. Genovese MC, McKay JD, Nasonov EL, et al. Interleukin-6 receptor inhibition with tocilizumab reduces disease activity in rheumatoid arthritis with inadequate response to disease-modifying antirheumatic drugs: the tocilizumab in combination with traditional disease-modifying antirheumatic drug therapy study. Arthritis Rheum. 2008;58:2968-80.

33. Emery P, Keystone E, Tony HP, et al. IL-6 receptor inhibition with tocilizumab improves randomised placebo-controlled trial biologicals: results from a 24-week multicentre arthritis refractory to anti-tumour necrosis factor treatment outcomes in patients with rheumatoid. Ann Rheum Dis. 2008;67:1516-23.

34. Charles P, Elliott MJ, Davis D, et al. Regulation of cytokines, cytokine inhibitors, and acute-phase proteins following anti-TNFalpha therapy in rheumatoid arthritis. J Immunol. 1999;163:1521-8.

35. Butler DM, Maini RN, Feldmann M, et al. Modulation of proinflammatory cytokine release in rheumatoid synovial membrane cell cultures: comparison of monoclonal anti-TNF-alpha antibody with the IL-1 receptor antagonist. Eur Cytokine Netw. 1995;6: 225-30.

36. Smolen JS, Aletaha D, Koeller M, et al. New therapies for treatment of rheumatoid arthritis. Lancet. 2007;370:1861-74.

37. Kishimoto T. The biology of interleukin-6. Blood. 1989;74:1-10.

38. Anderson JJ, Wells G, Verhoeven AC, Felson DT. Factors predicting response to treatment in rheumatoid arthritis: the importance of disease duration. Arthritis Rheum. 2000;43:22-9.

39. Nakamura I, Omata Y, Naito M, Ito K. Blockade of interleukin 6 signaling induces marked neutropenia in patients with rheumatoid arthritis. J Rheumatol. 2009;36:459-60.

40. Takeuchi T, Tatsuki Y, Nogami Y, et al. Postmarketing surveillance of the safety profile of infliximab in 5000 Japanese patients with rheumatoid arthritis. Ann Rheum Dis. 2008;67:189-94. 